Service social

\title{
Les comportements sexuels problématiques chez les enfants : pistes de réflexion pour une pratique en émergence
}

\section{Annick St-Amand, Marie-Pierre Villeneuve et Annie Vaillancourt}

Volume 63, numéro 1, 2017

Interventions en matière d'agressions sexuelles

URI : https://id.erudit.org/iderudit/1040031ar

DOI : https://doi.org/10.7202/1040031ar

Aller au sommaire du numéro

Éditeur(s)

École de service social de l’Université Laval

ISSN

1708-1734 (numérique)

Découvrir la revue

Citer cet article

St-Amand, A., Villeneuve, M.-P. \& Vaillancourt, A. (2017). Les comportements sexuels problématiques chez les enfants : pistes de réflexion pour une pratique en émergence. Service social, 63(1), 55-72. https://doi.org/10.7202/1040031ar
Résumé de l'article

Les comportements sexuels problématiques des enfants sont complexes et ont des répercussions sur plusieurs plans. Depuis les trente dernières années, des chercheurs ont tâché d'identifier les principaux facteurs de risque qui leur sont associés. Or, il n’y a pas de définition précise de ces comportements et les critères utilisés pour juger de leur caractère problématique sont variables. De nombreux professionnels affirment manquer de connaissance et de formation pour intervenir adéquatement. S’appuyant sur les résultats d'une méta-analyse et de recherches phares dans le domaine, cet article propose des pistes de réflexion afin d'alimenter cette pratique en émergence. 


\title{
Les comportements sexuels problématiques chez les enfants : pistes de réflexion pour une pratique en émergence
}

\author{
ST-AMAND, Annick \\ VILLENEUVE, Marie-Pierre \\ VAILLANCOURT, Annie
}

\begin{abstract}
RÉSUMÉ
Les comportements sexuels problématiques des enfants sont complexes et ont des répercussions sur plusieurs plans. Depuis les trente dernières années, des chercheurs ont tâché d'identifier les principaux facteurs de risque qui leur sont associés. Or, il n'y a pas de définition précise de ces comportements et les critères utilisés pour juger de leur caractère problématique sont variables. De nombreux professionnels affirment manquer de connaissance et de formation pour intervenir adéquatement. S'appuyant sur les résultats d'une méta-analyse et de recherches phares dans le domaine, cet article propose des pistes de réflexion afin d'alimenter cette pratique en émergence.
\end{abstract}

Mots clés : comportements sexuels problématiques, enfants, pratiques émergentes, intervention.

\begin{abstract}
Child sexual behavior problems are complex and have an impact on many levels. For the past thirty years, researchers have tried to identify the main risk factors associated with them. However, there is no precise definition of these behaviours and criteria to judge their problematic nature are variable. Many professionals claim lack of knowledge and training to respond appropriately. Based on the results of a meta-analysis and core researches in the field, this article aims to reflect on the characteristics of these children and intervention programs deemed effective to support this emerging practice.
\end{abstract}

Keywords: sexual behavior problems, children, emerging practices, intervention. 


\section{INTRODUCTION}

Les comportements sexuels problématiques (CSP) ${ }^{1}$ survenant entre enfants ont longtemps été ignorés ou considérés comme des jeux exploratoires anodins. On reconnaît aujourd'hui leurs effets néfastes sur les jeunes qui les manifestent, les enfants qui en sont témoins ou victimes, de même que sur les parents (biologiques, d'accueil ou adoptifs) ${ }^{2}$ et les intervenants qui les soutiennent (Baker, Schneiderman et Parker, 2001; Friedrich, Baker, Parker, Schneiderman, Gries et Archer, 2005; Shevade, Norris et Swann, 2011). Comme d'autres problèmes comportementaux, les CSP sont susceptibles de persister et de s'aggraver s'ils ne font pas l'objet d'une intervention adéquate (Chaffin et al., 2006).

À ce jour, il n'existe pas de définition opérationnelle des CSP qui fasse consensus. Plusieurs organismes d'aide à l'enfance ne disposent pas de définition précise (Baker, Schneiderman et Parker, 2001; Baker, Gries, Schneiderman, Parker, Archer et Friedrich, 2008; Johnson et Aoki, 1993) et les critères utilisés par les intervenants pour juger de leur caractère problématique semblent variables (Boisvert, 2016; Durand, 2006). Après analyse, il appert que les interventions sont souvent mises en place de manière aléatoire et subjective par les intervenants (Gil et Shaw, 2013). De nombreux professionnels affirment manquer de connaissance et de formation pour intervenir adéquatement (Baker, Schneiderman et Parker, 2001; Durand, 2006; Vosmer, Hackett et Callanan, 2009) et se sentir impuissants et tristes devant ces enfants (Durand, 2006; Shevade, Norris et Swann, 2011). Par ailleurs, si la sexualité est omniprésente dans notre environnement, elle demeure néanmoins un sujet tabou, délicat, voire gênant à aborder. Le fait que les adultes (parents, intervenants et autres pourvoyeurs de soins) ressentent souvent un inconfort à parler de sexualité infantile peut nuire à l'intervention et engendrer une réponse inappropriée ou inefficace aux besoins des enfants (Gil et Shaw, 2013).

Cet article vise à susciter la réflexion concernant les pratiques émergentes auprès des enfants manifestant des CSP, à la lumière des résultats d'une méta-analyse (St-Amand, Bard et Silovsky, 2008) et des travaux phares réalisés dans le domaine. L'objectif étant de mettre l'accent non seulement sur les interventions jugées efficaces et les éléments contribuant le plus activement à leur réussite, mais également sur les caractéristiques des personnes qui en bénéficient.

\section{Qui sont ces enfants?}

Selon un groupe de travail de l'Association for the Treatment of Sexual Abusers (ATSA), le phénomène des enfants manifestant des CSP concerne « des enfants âgés de 12 ans et moins qui initient des comportements impliquant des parties sexuelles du corps, qui sont inappropriés sur le plan développemental ou potentiellement néfastes pour eux-mêmes ou les autres » (Chaffin et al., 2006, p. 3, traduction libre). Ces comportements peuvent être dirigés vers l'enfant lui-même (ex. masturbation excessive) ou vers autrui. Les intentions et motivations de ces enfants ne sont pas nécessairement liées à la gratification sexuelle. Elles peuvent, entre autres, être associées à des sentiments d'anxiété ou de colère, à une expérience traumatisante, à une recherche

\footnotetext{
1 Afin d'alléger ce texte, l'acronyme CSP est utilisé en remplacement des termes comportements sexuels problématiques.

${ }^{2}$ Le terme parents réfère aux principaux dispensateurs de soins aux enfants, incluant les parents biologiques, adoptifs ou de familles d'accueil.
} 
d'attention ou à l'imitation de comportements sexuels observés dans l'environnement (Silovsky et Bonner, 2003).

Aucune étude de population ne permet de statuer sur la prévalence des enfants manifestant des CSP (Boisvert, 2016). Les intervenants des services de protection de l'enfance new-yorkais estiment qu'entre $10 \%$ et $30 \%$ des enfants bénéficiant de ces services présentent des CSP (Baker, Schneiderman et Parker, 2001). Au Québec, cette proportion est évaluée à $10 \%$, de même que pour l'ensemble des autres provinces canadiennes (Hélie, Turcotte, Trocmé et Tourigny, 2012). Les enfants manifestant des CSP présentent davantage de difficultés comportementales (comportements extériorisés et intériorisés problématiques, comportements délinquants, comportements agressifs), de symptômes liés au stress post-traumatique (reviviscences de l'événement, isolement social, difficultés cognitives) et de déficits sur le plan des compétences sociales (manque d'empathie, intensité et contrôle de la colère, évaluation négative des autres) que les enfants ne manifestant pas de CSP (Boisvert, Tourigny, Lanctôt et Lemieux, 2016). Par ailleurs, selon une recension systématique des écrits $(n=18)$ portant sur les facteurs associés aux CSP, en excluant les six études dont les échantillons sont composés uniquement d'enfants agressés sexuellement, entre $12 \%$ et $70 \%$ des enfants qui présentent des CSP ont également été victimes d'agression sexuelle (Boisvert, Tourigny, Lanctôt et Lemieux, 2016).

Le développement des CSP semble emprunter des trajectoires multiples et complexes (Chaffin, Letourneau et Silovsky, 2002), révélant une variété de facteurs de risque individuels (ex. déficits cognitifs et langagiers, troubles du comportement), relatifs à des histoires de maltraitance (ex. témoin de violence conjugale, victime d'agressions sexuelles, d'abus physiques, de mauvais traitements psychologiques) ou à l'environnement familial (ex. abus de substances ou détresse psychologique d'un parent, manque d'encadrement ou de supervision, environnement familial plus sexualisé, revenus précaires) (Boisvert et al., 2016; Friedrich, Davis, Fehrer et Wright, 2003). Bien qu'il existe des processus interactifs entre ces différents facteurs de risque (Friedrich, 2007), il est difficile d'établir l'importance de chacun ou de se prononcer sur les combinaisons de facteurs ayant le plus d'impact. Aucun profil-type ne permet de distinguer les enfants manifestant des CSP des autres groupes d'enfants (Chaffin, Letourneau et Silovsky, 2002). La multiplicité et la complexité des trajectoires pourraient expliquer en partie les différences en matière de risque et de pronostic, particulièrement considérables d'un stade de développement à l'autre (Chaffin, Letourneau et Silovsky, 2002; Elkovitch, Latzman, Hansen et Flood, 2009).

Les difficultés vécues par ces enfants et leur famille augmentent le risque qu'ils soient séparés de leur famille d'origine et perturbés par ce placement ${ }^{3}$ (Baker, Schneiderman et Parker, 2001; Boisvert et al., 2016; Bonner, Walker et Berliner, 1999; Silovsky et Niec, 2002). Ils sont plus susceptibles d'être déplacés d'une famille d'accueil à l'autre, puisque les adultes qui en sont responsables ne disposent pas d'outils adéquats pour composer avec leurs difficultés (Baker et al., 2008; Friedrich et al., 2005). Le portrait clinique des enfants ayant été victimes de maltraitance est souvent plus complexe en raison de la manifestation de symptômes liés à ces traumatismes (ex. symptômes de dépression, anxiété et syndrome de stress post-traumatique) (Boisvert et al.,

\footnotetext{
${ }^{3}$ Lorsque les enfants présentant des CSP sont retirés de leur milieu familial, les interventions doivent être adaptées afin d'inclure leurs principaux dispensateurs de soins et les autres acteurs impliqués dans leur milieu de vie.
} 
2016; Bonner, Walker et Berliner, 1999; Pithers et Gray, 1998; Silovsky et Niec, 2002). La prise en compte de ces symptômes constitue un défi supplémentaire en intervention.

\section{À partir de quand peut-on parler de comportements sexuels problématiques ?}

Puisqu'il n'existe aucune définition opérationnelle claire des CSP à l'heure actuelle (Lepage, Tourigny, Pauzé, McDuff et Cyr, 2010; Szanto, Lyons et Kisiel, 2012), il n'y a pas de limite précisant le moment à partir duquel un comportement sexuel, en apparence anodin, doit être considéré comme anormal ou malsain (Johnson, 2002). Cette distinction est d'autant plus difficile à faire qu'elle s'appuie généralement sur des considérations normatives (âge et stade de développement de l'enfant) (Szanto, Lyons et Kisiel, 2012) et culturelles (Chaffin, Letourneau et Silovsky, 2002). Selon plusieurs experts (Araji, 1997; Chaffin et al., 2006; Hall, Mathews et Pearce, 1998; Johnson, 2004), différents indicateurs devraient être considérés pour juger du caractère problématique des comportements sexuels : a) ils surviennent à une fréquence élevée; b) ils sont devenus une préoccupation importante pour l'enfant; c) ils persistent malgré l'intervention d'un adulte; d) ils surviennent entre des enfants d'âge, de taille ou de stade développemental différents; e) ils se manifestent par le recours à la force, à la coercition ou à l'intimidation; f) ils sont associés à une détresse émotionnelle chez l'enfant (les enfants) impliqué(s); g) ils interfèrent dans le développement social de l'enfant (des enfants); ou h) ils entraînent des blessures physiques.

Les CSP varient considérablement selon leur nature, leur intensité et leur sévérité. L'examen des différentes classifications cliniques disponibles à ce jour dans la littérature permet de proposer un continuum, des moins problématiques aux plus critiques : 1) les comportements sexuellement inappropriés sans contacts interpersonnels, qu'ils soient dirigés vers soi-même (ex. masturbation causant des blessures) ou autrui (ex. utilisation constante de mots sexuels en présence d'autrui) (Hall, Mathews, Pearce, Sarlo-McGarvey et Gavin, 1996; Silovsky et Swisher, 2008); 2) les comportements sexuellement intrusifs, comportant des contacts interpersonnels non planifiés, impulsifs et non agressifs (Bonner, Walker et Berliner, 1999; Hall et al., 1996); et 3) les comportements sexuellement agressifs, comprenant des contacts sexuels caractérisés par un recours aux menaces, à la force, à la coercition ou à l'agression (Berliner, Manaois et Monastersky, 1986; Bonner, Walker et Berliner, 1999; Hall et al., 1996; Hall, Mathews et Pearce, 1998; Silovsky et Swisher, 2008). Ces derniers sont les plus inquiétants, surtout s'ils se produisent entre enfants présentant une grande différence d’âge (Johnson, 2009).

\section{Précisions méthodologiques}

Cette réflexion entourant les pratiques émergentes auprès des enfants manifestant des CSP résulte de l'examen critique d'un bassin de travaux. Une portion de ces travaux ont été localisés dans le cadre de la méta-analyse de St-Amand, Bard et Silovsky (2008) ${ }^{4}$. Dans cette recherche,

\footnotetext{
${ }^{4}$ Cette méta-analyse porte sur 11 études rapportant les résultats obtenus au terme de 18 interventions spécifiques ciblant les CSP (cible primaire) ou les effets de l'abus sexuel incluant le développement de CSP (CSP comme cible secondaire). Chacune de ces interventions a fait l'objet d'un examen approfondi à l'aide des manuels officiels de traitement et des résultats obtenus au Child Sexual Behavior Inventory (CSBI) et au Child Behavior Checklist (CBCL), avant et après la participation de l'enfant à celles-ci. Pour de plus amples détails concernant la méthodologie de cette étude, le lecteur est invité à consulter l'article publié en 2008.
} 
plusieurs bases de données électroniques pertinentes au domaine d'étude ont été interrogées (Current contents, Education abstracts, ERIC, Familia, Family \& society studies worldwide, Francis, MEDLINE, Psychology and behavioral sciences collection, PsyclNFO, ScienceDirect, Social services abstracts, Social work abstracts et Sociological abstracts) à partir d'une stratégie assurant une recherche judicieuse et efficace ${ }^{5}$. La liste de références de tous les documents consultés a été systématiquement examinée, incluant celle de volumes pertinents. Cette stratégie de recherche a été répétée pour la période allant de 2007 à 2015 de manière à repérer les travaux non recensés par la méta-analyse, dont la période de couverture allait jusqu'en 2006.

\section{Quelques constats relatifs aux pratiques en cours}

Pour répondre aux fortes préoccupations sociales et aux besoins criants des milieux de pratique concernés par le phénomène des enfants aux CSP, des interventions ont vu le jour en Amérique du Nord et ailleurs au cours des trente dernières années : des pratiques singulières, développées par des praticiens privés, aux programmes structurés à grande échelle, offerts par des agences de services impliquant plusieurs praticiens. Cependant, peu de ces interventions ont fait l'objet d'une évaluation empirique de leur efficacité et les études évaluatives existantes présentent des limites méthodologiques. Quoi qu'il en soit, les résultats de ces études alimentent le bassin de connaissances relatives au phénomène des CSP ainsi qu'aux caractéristiques des interventions prometteuses et permettent d'établir certains constats afin d'alimenter la réflexion entourant cette pratique en émergence.

Premièrement, on observe généralement une amélioration des CSP à la fin des interventions (Chaffin et al., 2006; St-Amand, Bard et Silovsky, 2008), mais les devis des recherches évaluatives ne permettent pas de savoir si cette amélioration est due aux traitements ou à des facteurs externes (Gagnon et Tourigny, 2011). Peu d'études contrôlées randomisées ont été réalisées. L'évaluation de l'efficacité porte donc sur les caractéristiques des enfants et des programmes d'intervention associées à une diminution des CSP, plutôt que sur l'impact de bénéficier ou non de l'intervention. Les évaluations sont basées sur les résultats obtenus à deux tests standardisés (CSBI et CBCL) et ne prennent pas en compte les multiples facteurs (ex. le passage du temps) pouvant influencer les effets observés (St-Amand et al., 2008). Deuxièmement, les interventions axées sur les CSP obtiennent des résultats positifs, ce qui semble particulièrement vrai lorsqu'elles sont structurées, font participer les parents (ou principaux dispensateurs de soins) et se fondent sur une approche cognitive-comportementale (Chaffin et al., 2006; Gagnon et Tourigny, 2011). Troisièmement, une méta-analyse (St-Amand, Bard et Silovsky, 2008) révèle que : a) les interventions d'approche cognitive-comportementale qui se centrent à la fois sur les CSP et les symptômes de stress post-traumatique peuvent contribuer à diminuer ces deux problèmes lorsqu'ils sont présents; b) les interventions les plus profitables sur le plan de la réduction des CSP sont celles qui misent sur l'éducation et l'enseignement de règles et d'habiletés auprès des parents (ou principaux dispensateurs de soins) et qui permettent, dans une certaine mesure, de réunir les membres pour une intervention familiale; c) les interventions, qui s'adressent aux enfants d'âge préscolaire et misent sur

\footnotetext{
${ }^{5}$ Les termes suivants ont été utilisés dans les champs titre et mots-clés: (sexually reactive child* OU sexually aggressive child* OU child perpetrator OU child offender OU child molester OU harmful sexual behavior OU sexual behavior problem) ET (therap* OU psychotherap* OU treatment OU program, OU intervention OU outcome).
} 
l'enseignement d'habiletés parentales de gestion des comportements, sont celles qui produisent les changements positifs de plus grande ampleur dans les CSP des enfants; et d) le fait d'aborder des thèmes empruntés aux interventions pour adultes auteurs d'agression sexuelle (ex. le cycle de l'abus/du CSP, l'excitation sexuelle et le reconditionnement, la compréhension des impacts des CSP et les excuses) ne contribue pas à une réduction plus importante des CSP.

À la lumière de ces constats et des connaissances actuelles, la suite de cet article amorce une réflexion concernant les orientations à privilégier en intervention auprès des enfants et des familles touchées par cette problématique. Sur quelles assises pouvons-nous nous appuyer pour élaborer des interventions adaptées aux besoins de cette clientèle?

\section{PISTES DE RÉFLEXION}

\section{L'implication des figures parentales comme acteurs de soutien au changement}

Un premier élément de réflexion a trait au rôle clé que semblent jouer les parents ou les principaux dispensateurs de soins non abuseurs en tant qu'acteurs de soutien au changement (voir Cohen, Mannarino et Deblinger, 2006; Silovsky, Swisher, Widdifield et Burris, 2012). Cette première piste peut sembler paradoxale, puisque certaines caractéristiques de l'environnement familial sont impliquées dans l'étiologie des CSP (voir Chaffin et al., 2006; Chaffin, Letourneau et Silovsky, 2002). Or, l'implication des parents non abuseurs dans l'intervention doit être considérée afin d'agir sur ces facteurs de risque (Chaffin et al., 2006; Cohen, Mannarino et Deblinger, 2006) et sur les stresseurs pouvant nuire à la capacité des parents à fournir les soins optimaux à leur enfant (voir Friedrich, 2007). La détresse émotionnelle des parents peut nuire, entre autres, à l'exercice de leur rôle parental et au soutien à l'enfant dans la mise en application des apprentissages réalisés au cours de l'intervention (Silovsky et al., 2012). Une recension systématique des écrits portant sur les facteurs associés aux CSP chez les enfants de 12 ans et moins conclut à la nécessité de considérer à la fois les caractéristiques de l'enfant et celles de son environnement familial dans la pratique psychosociale et de favoriser le développement de saines relations parents-enfant (Boisvert et al., 2016).

Les résultats appuyant cette première piste de réflexion remettent en question les interventions actuelles qui considèrent comme prépondérants les facteurs relatifs à l'enfant dans l'origine et le maintien des CSP et qui, par conséquent, se concentrent exclusivement (ou presque) sur les déficits ou difficultés des enfants et contribuent à la «pathologisation » de ces comportements. Cela questionne également les interventions qui prennent place en milieu hospitalier ou en internat de réadaptation, sans impliquer significativement les figures parentales (ou principaux dispensateurs de soins) à un moment ou l'autre du processus d'intervention.

L'implication parentale a maintes fois été identifiée comme une variable fortement associée à l'efficacité des interventions auprès des enfants manifestant des problèmes de comportement (voir Brestan et Eyberg, 1998; Deblinger, Steer et Lippman, 1999; Dretzke et al., 2009; Kazdin, 2005; Nixon, 2002). Dans le champ plus spécifique des CSP, la pratique psychosociale devrait s'inspirer du consensus émergeant en Australie, en Grande-Bretagne, aux États-Unis, en France et au Québec, quant à l'importance d'impliquer activement les parents dans le processus d'intervention (Evertsz et al., 2010; Friedrich, 2007; Gagnon, Tourigny et Lévesque, 2008; 
Hackett, 2011; Silovsky, Niec, Bard et Hecht, 2007; Silovsky et al., 2012). Ce consensus fait écho à la reconnaissance grandissante de l'influence des variables parentales et familiales dans l'étiologie des CSP (Lepage et al., 2010). Notamment, l'environnement sexualisé qui semble caractériser un nombre non négligeable de familles (Boisvert et al., 2016) peut référer à l'absence de frontières interpersonnelles, à l'accessibilité à du matériel de nature sexuelle ou pornographique, ou encore à l'exposition à la nudité et à des activités sexuelles d'adultes. La présence de ces caractéristiques dans l'environnement familial pourrait, en partie, expliquer la contribution marquée de certaines composantes à la réussite de l'intervention. En effet, l'apprentissage et l'application de règles concernant les comportements sexuels, la sensibilisation aux manières appropriées d'entrer en relation et au respect de l'intimité et des frontières de chacun, de même que l'apprentissage de moyens efficaces pour assurer la supervision et la protection des enfants pourraient s'avérer particulièrement bénéfiques pour l'ensemble des membres de ces familles.

Dans la mesure où les enfants sont ancrés dans leur famille et qu'ils agissent, bien souvent, en fonction des expériences qu'ils ont vécues au sein de cet environnement (Friedrich, 2007), il est primordial que les pratiques visent à le modifier en faisant du système parents-enfant la pierre angulaire de l'intervention (Etgar et Shulstain-Elrom, 2010). Dans la plupart des cas, le parent impliqué risque d'être l'acteur qui va assurer le transfert des apprentissages, au sein de la famille notamment, et qui va favoriser le maintien des acquis chez l'enfant. Par ailleurs, dans les situations où l'environnement ne contribue pas au problème, l'implication des parents apparaît tout aussi importante de manière à assurer un soutien à l'enfant et la mise en application des éléments du plan d'intervention qui relèvent du quotidien (Chaffin et al., 2006). L'implication parentale peut prendre différentes formes : participation à des sessions d'intervention en parallèle des sessions tenues avec l'enfant (en groupe ou en individuel), sessions conjointes où parents et enfants sont réunis pour partager ou mettre en pratique leurs apprentissages (en groupe ou individuellement) ou interventions dans le milieu de vie de l'enfant. II est recommandé de diriger les parents vers une ressource appropriée lorsqu'ils ont eux-mêmes été victimes de mauvais traitements dans l'enfance, qu'ils ont vécu un traumatisme qui n'est pas résolu ou qu'ils présentent une grande détresse émotionnelle (Cohen et al., 2006).

\section{Le rôle central des habiletés parentales de gestion des comportements}

Le fait que l'enseignement d'habiletés parentales de gestion des comportements se soit avéré l'ingrédient le plus efficace dans la réduction des CSP, selon une méta-analyse (St-Amand, Bard et Silovsky, 2008), appuie encore plus fortement le développement de pratiques où les parents seraient des agents actifs. À l'instar de certains problèmes de comportement, les CSP pourraient être réduits plus facilement grâce à l'application constante et cohérente de stratégies parentales adéquates et appropriées au développement de l'enfant. Ainsi, l'intervenant devrait accompagner le parent dans le développement d'habiletés telles que de renforcer les comportements appropriés par les félicitations et les systèmes de récompenses, d'établir des limites et des consignes claires, ou d'utiliser adéquatement l'ignorance, le retrait, l'attention sélective et les autres conséquences logiques.

Certains facteurs de risque sont communs aux enfants présentant des problèmes de comportement extériorisés et aux jeunes manifestant des CSP. II y aurait donc lieu d'explorer la 
pertinence, pour les enfants présentant ces deux problématiques, de s'inspirer davantage des approches d'entraînement aux habiletés parentales (ex. Parent-Child Interaction Therapy Brestan et Eyberg, 1998; The Incredible Years - Webster-Stratton, 2005; Barkley's Defiant Child protocole - Barkley et Benton, 1998; Triple-P program - Sanders, Cann et Markie-Dadds, 2003), qui ont à maintes reprises démontré leur efficacité à réduire les problèmes de comportement. Contrairement aux interventions qui ciblent les CSP, les programmes basés sur ces approches considèrent habituellement les habiletés de gestion des comportements comme centrales et y consacrent plusieurs sessions. En plus de travailler à la modification du comportement parental, ces approches visent le développement d'habiletés relationnelles favorables à l'établissement d'une bonne relation parent-enfant (ex. communiquer adéquatement avec l'enfant, interagir positivement avec lui).

Par ailleurs, dans la mesure où l'apprentissage d'habiletés de gestion des comportements s'accompagne presque toujours, dans le contexte des interventions ciblant les CSP, d'enseignements relatifs à l'éducation sexuelle, à la prévention des abus et aux règles concernant les comportements sexuels (St-Amand, Bard et Silovsky, 2008), il serait intéressant d'envisager une intervention qui s'inspirerait des approches d'entraînement aux habiletés parentales tout en intégrant des cibles plus spécifiques à la dimension sexuelle. Cela rejoindrait les recommandations d'un groupe d'experts (Chaffin et al., 2006), à l'effet que les pratiques visant les parents d'enfants aux CSP devraient favoriser l'enseignement d'habiletés de gestion des comportements, de même que l'établissement d'un plan de supervision et d'un environnement sécuritaire non sexualisé.

\section{Tenir compte des particularités développementales des enfants}

Un sondage nord-américain réalisé en 2009 par la Safer Society révèle que la majorité des programmes d'intervention destinés aux enfants ayant commis des gestes sexuels abusifs incluent des pratiques issues des modèles d'intervention pour adultes agresseurs sexuels (McGrath, Cumming, Burchard, Zeoli et Ellerby, 2010). Or, la légitimité de ces emprunts à des interventions développées pour des individus se situant à un stade développemental distinct est actuellement remise en question.

En effet, les résultats d'une méta-analyse suggèrent que le fait d'aborder des thèmes issus des interventions pour adolescents ou adultes auteurs d'agression sexuelle, tels le cycle de l'abus/du CSP, l'excitation sexuelle et le reconditionnement, la responsabilité vis-à-vis de l'offense ou la compréhension des impacts des CSP et les excuses, ne contribue pas à une réduction plus importante des CSP que le fait de ne pas les aborder (St-Amand, Bard et Silovsky, 2008). L'inclusion de l'une de ces cibles d'intervention serait même associée à une réduction moins importante des problèmes de comportement généraux chez les enfants. Par ailleurs, plusieurs auteurs insistent sur l'importance de développer des interventions en accord, tant dans leur structure que dans leur contenu, avec des particularités des clientèles appartenant à des niveaux développementaux distincts (Chaffin et al., 2006; Jaffé, 2011; Silovsky et al., 2012).

Selon Silvosky et al. (2012), les origines des CSP, les motivations à les initier et à les maintenir ainsi que la réponse des enfants à l'intervention sont distinctes de celles des adultes auteurs d'agression sexuelle, d'où l'importance de prendre en compte les aspects cognitifs et sociaux de 
leur développement pour assurer une réponse adéquate à leurs besoins. II est préférable d'enseigner des règles simples et concrètes aux enfants manifestant des CSP, par exemple, ne pas toucher les parties intimes des autres. Cet apprentissage se fera plus facilement si l'intervenant a recours à des stratégies telles l'observation, la mise en pratique et le renforcement (Chaffin et al., 2006). Les enfants sont en mesure de comprendre les « bons » et les « mauvais » comportements, ainsi que les conséquences immédiates (punition, renforcement) associées à ceux-ci (Silovsky et al., 2012). II peut donc être plus bénéfique de privilégier le modelage de comportements appropriés et le renforcement de leur application dans différents contextes (Chaffin et al., 2006), notamment par les jeux de rôle. Les rencontres avec les enfants doivent être structurées, routinières et répétées, tout en tenant compte de leur faible capacité attentionnelle (Silovsky et al., 2012).

La planification de l'intervention devrait considérer, en plus de l'âge de l'enfant, son niveau de développement sur les plans langagier, cognitif, moral et social. Les enfants n'évoluent pas tous au même rythme, puisque leur trajectoire développementale est influencée par leurs caractéristiques personnelles (ex. capacités cognitives), celles de leur environnement (ex. qualité de la relation parent-enfant, valeurs et normes véhiculées) et les événements qui ponctuent leur vécu (ex. histoire de mauvais traitements).

\section{Le choix d'une modalité d'intervention}

Plusieurs interventions ayant les CSP pour cible primaire ont recours à une modalité de groupe (St-Amand, Bard et Silovsky, 2008). Certains chercheurs et praticiens considèrent cette modalité d'intervention comme particulièrement bénéfique, mais ses avantages hypothétiques n'ont jamais fait l'objet de vérification empirique auprès d'enfants manifestant des CSP. Du côté des interventions d'entraînement aux habiletés parentales, peu d'études ont examiné l'efficacité relative des modalités de groupe et individuelle (voir Brightman, Baker, Clark et Ambrose, 1982; Pevsner, 1982; Webster-Stratton, 1984). Celles qui l'ont fait ont mené à des résultats inconsistants. II est possible que les avantages relatifs à la modalité de groupe soient liés à des dimensions autres que le CSP lui-même, comme le stress parental, le soutien des participants ou les habiletés sociales de l'enfant (Niec, Hemme, Yopp et Brestan, 2005).

Parmi les bénéfices souvent associés à l'intervention de groupe, notons : l'apprentissage par observation/imitation, la réduction du sentiment d'isolement, le développement d'habiletés sociales, des discussions plus actives ainsi que de faibles coûts pour les organisations (Chaffin et al., 2006). Tant pour les parents que l'enfant, le groupe permettrait d'éviter la stigmatisation de l'intervention en normalisant les situations vécues, d'aborder le comportement sexuel directement, puis de mettre en pratique les habiletés et comportements appris avec le soutien de l'intervenant et les rétroactions des pairs. De plus, l'intervention de groupe responsabilise les parents en ce qui a trait à la gestion des comportements de leur enfant (Silvosky et al., 2012). En général, la formation de groupes est mixte et tient compte de l'âge ainsi que des habiletés développementales des participants (souvent regroupés ainsi : 3-5 ans, 6-9 ans, 10-12 ans). L'intervenant devrait toutefois s'assurer de préserver la confidentialité des situations et de recourir à des stratégies efficaces de gestion des comportements avec les enfants participants. 
En complément, l'intervention individuelle auprès de l'enfant et de sa famille pourrait s'avérer utile pour aborder des problèmes concomitants et répondre à des besoins spécifiques (Chaffin et al., 2006; Silovsky et al., 2012) ou lorsqu'une intervention de crise est nécessaire (Silovsky et al., 2012). Dans les cas d'importants retards cognitifs ou de problèmes majeurs de santé mentale, et dans les cas de CSP n'impliquant pas d'autres enfants (ex. masturbation compulsive), les bénéfices de l'intervention individuelle (auprès de l'enfant et de sa famille) comme modalité unique sont relevés par certains (Gil, 1993), mais n'ont pas été vérifiés empiriquement. II peut être plus facile de recourir à cette modalité dans les communautés rurales, où le groupe semble plus menaçant, ou encore dans les milieux d'intervention où on rencontre moins de situations comprenant des enfants aux CSP (Chaffin et al., 2006). Enfin, dans le choix de la ou des modalités à privilégier, les praticiens devraient avant tout tenir compte des caractéristiques particulières de l'enfant et du contexte.

\section{Favoriser la flexibilité des interventions}

Les connaissances actuelles permettent, dans un premier temps, de statuer sur le portrait très hétérogène des enfants manifestant des CSP sur le plan des caractéristiques démographiques et familiales, de l'histoire de maltraitance, des problèmes concomitants et de la nature des comportements sexuels adoptés (Chaffin, Letourneau et Silovsky, 2002; Elkovitch et al., 2009). Elles permettent aussi, dans un deuxième temps, de penser que les CSP pourraient constituer un problème isolé, une réaction liée à un traumatisme, un symptôme issu d'un trouble du comportement ou d'une autre condition clinique préoccupante ou encore une combinaison de ces éléments (Silovsky et al., 2012). Conséquemment, un manque de flexibilité en intervention pourrait s'avérer particulièrement problématique.

À la lumière des données empiriques et des recommandations issues de la littérature, il apparaît pertinent pour les milieux de pratique d'envisager une intervention assortie aux particularités cliniques et sociodémographiques des enfants et de leur famille, plutôt que de privilégier une intervention standard pour tous les enfants (Boisvert, 2016; Chaffin et al., 2006; Gagnon, Tourigny et Lévesque, 2008; Silovsky et al., 2012). C'est d'ailleurs ce que prône la pratique fondée sur des données probantes qui consiste en " une intégration des meilleures preuves issues de la recherche, de l'expertise clinique et des valeurs et circonstances uniques du client » (Straus, Richardson, Glasziou et Haynes, 2011, p. 85, traduction libre). Pourtant, plusieurs experts estiment que la diversité des enfants qui manifestent des CSP a jusqu'à maintenant été sousestimée dans le développement d'interventions et de politiques publiques (Chaffin et al., 2006).

\section{L'intérêt d'une lecture bioécologique pour guider l'intervention}

À ce jour, les interventions destinées aux enfants manifestant des CSP s'inspirent majoritairement des modèles du trauma, des théories de l'apprentissage social et des systèmes familiaux ainsi que de la théorie de l'attachement pour expliquer l'origine de ces comportements (St-Amand, Saint-Jacques et Silovsky, 2011). Pour bien saisir l'apport de ces diverses perspectives théoriques dans l'explication spécifique des CSP, Friedrich (2007) propose une adaptation du modèle théorique de Greenberg, Speltz et Deklyen (1993), développé pour expliquer l'origine des comportements perturbateurs. Son modèle intégrateur repose sur les 
interactions entre quatre domaines de risque. Récemment, pour pallier certaines lacunes du modèle de Friedrich, Boisvert et ses collaborateurs (2016) proposent à leur tour une adaptation plus fidèle du modèle de Greenberg, Speltz et Deklyen, (1993) qui conçoit le développement des CSP à travers les interactions entre cinq domaines de risque : 1) les facteurs individuels prédisposant aux problèmes de comportement; 2) les stresseurs affectant la capacité des parents à fournir des soins optimaux à leur enfant; 3) les pratiques parentales coercitives; 4) la perturbation du développement psychosexuel de l'enfant; et 5) la qualité de l'attachement parentenfant. Or, la façon dont ces domaines de risque interagissent ainsi que le poids relatif de chacun d'eux demeurent méconnus.

À la lumière des pistes de réflexion soulevées jusqu'ici, il semble que le développement des interventions ciblant les CSP doit prendre appui sur une lecture nuancée de la problématique, qui permet de bien saisir la complexité des situations vécues par ces enfants et leur famille. À ce titre, l'approche bioécologique (Bronfenbrenner, 1979; Bronfenbrenner et Morris, 2006; Bruyère et Garbarino, 2010) apparaît particulièrement intéressante pour l'évaluation des CSP puisqu'elle situe l'adaptation comme le résultat de l'interaction et de l'interinfluence entre plusieurs éléments contextuels proximaux (situés dans les contextes de vie immédiats) et distaux (situés dans des sphères plus éloignées) qui se modifient au fil du temps. L'approche reconnaît qu'au fur et à mesure que l'individu se développe et vieillit, de nouvelles sources d'influence interviennent sur le plan développemental. Conformément à ses principes fondamentaux, l'approche bioécologique stipule que : 1) l'enfant est un individu qui contribue à modeler sa trajectoire développementale, mais que son développement se produit « en contexte »; 2) les processus relationnels proximaux dans les milieux de vie de l'enfant (ex. relations parents-enfant, frère/sœur-enfant, enseignant-enfant, pairs-enfant) constituent les moteurs de son développement; et 3) les forces sociales et économiques qui s'exercent sur les milieux de vie immédiats affectent la qualité des processus qui s'y déroulent. Ces trois principes permettent une compréhension plus complète de la manière dont surviennent et s'aggravent les CSP des enfants et, en conséquence, pourraient contribuer au développement d'une pratique adaptée à leurs besoins spécifiques.

Les résultats obtenus par St-Amand, Bard et Silovsky (2008) soulignent, d'une part, l'importance de travailler sur les difficultés et à partir des capacités des parents et de la famille et, d'autre part, l'importance de considérer tous les aspects développementaux au moment de la planification des interventions. II reste néanmoins fort à faire pour saisir la complexité des CSP d'un point de vue écologique et traduire cette compréhension en intervention efficace, car les facteurs distaux ou certains niveaux de système sont beaucoup moins étudiés à ce jour. II est désormais reconnu que les risques associés aux CSP relèvent de sphères d'influence multiples. II importe donc de favoriser une évaluation et une intervention holistiques (Gagnon, Tourigny et Lévesque, 2008; Jaffé, 2011). Par contre, l'influence des sphères économique (ex. pauvreté au sein de la communauté), sociale (ex. culture dominante) et développementale (ex. résolution des tâches développementales) a fait l'objet de moins d'attention et doit être investiguée davantage pour assurer une réponse optimale de la part des services psychosociaux.

Dans cet ordre d'idées, certains auteurs soulignent qu'en intervention, différents environnements doivent être considérés pour offrir une supervision et un encadrement adéquats à l'enfant manifestant des CSP, assurer le respect des règles relatives à la sécurité et à l'intimité et susciter 
le développement d'interactions prosociales avec ses pairs (Chaffin et al., 2006; Silovsky et al., 2012). Dans cette optique, ils soulèvent la possibilité d'impliquer d'autres adultes (ex. enseignants ou éducateurs à la petite enfance) ou pairs significatifs dans le réseau social de l'enfant, soit en intervention, soit dans l'établissement d'un plan de protection, à condition de considérer des enjeux tels que le respect de la confidentialité et de la vie privée et de tenir compte des préjugés pouvant être entretenus à l'égard de l'enfant. Cette recommandation pourrait s'avérer particulièrement intéressante pour les enfants d'âge scolaire qui passent plus de temps à l'extérieur de leur sphère familiale, intègrent d'autres lieux de socialisation et côtoient d'autres agents de renforcement que leurs parents. L'implication du milieu scolaire et du groupe de pairs peut permettre d'accroître les facteurs de protection dans l'environnement de l'enfant, en favorisant le développement d'amitiés positives, en augmentant le soutien social qui lui est offert, en améliorant ses habiletés de communication, en l'aidant à développer des frontières saines et en lui enseignant des stratégies d'adaptation adéquates. La participation de tiers en tant qu'acteurs complémentaires (en soutien à l'enfant et à sa famille) peut favoriser l'apprentissage et le développement d'habiletés (ex. relationnelles, de résolution de problèmes) et renforcer des comportements appropriés ou prosociaux dans différents contextes de vie.

\section{CONCLUSION}

Cet article visait à susciter une réflexion sur l'intervention à privilégier auprès d'enfants manifestant des CSP. Les connaissances sur ce phénomène se sont particulièrement développées au cours des trente dernières années, mais il reste encore fort à faire pour cerner les meilleures pratiques à adopter en intervention. Néanmoins, plusieurs études nous renseignent sur les nombreux facteurs de risque à considérer, ainsi que sur la diversité et la complexité des trajectoires développementales des CSP. Il est désormais admis que les CSP sont associés à un amalgame de facteurs de risque individuels, développementaux, familiaux et environnementaux, et que leurs conséquences touchent non seulement l'enfant qui les manifeste, mais aussi la victime, l'entourage respectif des enfants concernés par cette problématique (famille, amis, enseignants) et les professionnels appelés à les soutenir.

Malgré leurs limites méthodologiques, les études ayant évalué l'efficacité des interventions ciblant les CSP, comme cible primaire ou secondaire, ont permis de dégager des pistes de réflexion pour guider cette pratique en émergence. L'implication des parents non abuseurs (ou principaux pourvoyeurs de soins) paraît incontournable, de même que l'enseignement d'habiletés parentales de gestion des comportements (permettant de réduire les CSP) et un contexte d'intervention offrant à l'enfant un environnement sécuritaire, favorisant le développement de comportements prosociaux et de comportements sexuels adéquats. Il est aussi possible d'intervenir directement auprès de l'enfant, dans la mesure où les stratégies et les contenus privilégiés tiennent compte de ses capacités cognitives et sociales. La modalité retenue peut être autant individuelle que familiale ou de groupe, ou une combinaison de ces trois formules. L'important est d'orienter ses décisions en fonction d'une évaluation holistique de la situation, afin d'adapter l'intervention aux besoins spécifiques du jeune et de sa famille. À cet effet, il apparaît donc particulièrement pertinent de procéder à une lecture bioécologique de chaque situation.

Pour continuer d'alimenter la réflexion sur les pratiques favorables aux enfants manifestant des CSP et leur famille, il est impératif d'intensifier les efforts en recherche. Ces derniers doivent 
reposer sur des devis rigoureux permettant d'appuyer davantage les constats actuels et de proposer de nouvelles avenues. II va sans dire que l'établissement d'une définition opérationnelle et consensuelle des CSP constitue une étape fondamentale à la poursuite des travaux. Cela permettrait de construire un corpus de connaissances empiriques et pratiques applicables dans une pluralité de contextes. En bout de piste, ce sont les enfants et leur famille qui en sortiraient gagnants. Les intervenants seraient mieux outillés pour prévenir et dépister les CSP, limitant ainsi le nombre de victimes potentielles et les conséquences psychosociales qui en découlent. Investir en recherche dans ce domaine d'intervention pourrait donc réduire les coûts sociaux associés à cette problématique.

Annick St-Amand, Ph.D. service social, M. Serv. soc. Psychoéducatrice et professeure agrégée Département de psychoéducation Université du Québec à Trois-Rivières - Centre universitaire de Québec

Marie-Pierre Villeneuve, M. Serv. soc. Candidate au doctorat en service social Université Laval

Annie Vaillancourt, M. Serv. soc.

Agente de planification, de programmation et de recherche Centre intégré universitaire de santé et de services sociaux de la Capitale Nationale

\section{RÉFÉRENCES}

Araji, S.K. (1997). Sexually aggressive children: Coming to understand them, Thousand Oaks (CA), Sage.

Baker, A.J.L., L. Gries, M. Schneiderman, R. Parker, M. Archer et B. Friedrich (2008). « Children with problematic sexualized behaviors in the child welfare system », Child Welfare, vol. 87, $\mathrm{n}^{\circ}$ 1, p. 5-27.

Baker, A.J.L., M. Schneiderman et R. Parker (2001). " A survey of problematic sexualized behaviors of children in the New York City child welfare system: Estimates of problem, impact on services, and need for training ", Journal of Child Sexual Abuse, vol. 10, n 4, p. 67-80.

Barkley, R., et C. Benton (1998). Your defiant child: Eight steps to better behavior, New York (NY), Guilford Press.

Berliner, L., O. Manaois et C. Monastersky (1986). Child sexual behavior disturbance: An assessment and treatment model, Seattle (WA), Harborview Sexual Assault Center. 
Boisvert, I. (2016). Approfondissement du lien entre l'agression sexuelle et les comportements sexuels problématiques chez les enfants de 12 ans et moins, Thèse de doctorat, Faculté d'éducation, Université de Sherbrooke (QC).

Boisvert, I., M. Tourigny, N. Lanctôt et S. Lemieux (2016). " Comportements sexuels problématiques chez les enfants: une recension systématique des facteurs associés ", Revue de psychoéducation, vol. 45, $\mathrm{n}^{\circ} 1, \mathrm{p} .173-208$.

Bonner, B.L., C.E. Walker et L. Berliner (1999). Children with sexual behavior problems: Assessment and treatment. Final report (Grant No. 90-CA-1469), US Department of Health and Human Services, National Clearinghouse on Child Abuse and Neglect.

Brestan, E.V., et S.M. Eyberg (1998). « Effective psychosocial treatments of conduct disordered children and adolescents: 29 years, 82 studies, and 5,272 kids », Journal of Clinical Child Psychology, vol. 27, n², p. 180-189.

Brightman, R.E, B.L. Baker, D.B. Clark et S.A. Ambrose (1982). « Effectiveness of alternative parent training formats ", Journal of Behavior Therapy and Experimental Psychology, vol. 13, $n^{\circ} 2$, p. $113-117$.

Bronfenbrenner, U. (1979). The ecology of human development: Experiments by nature and design, Cambridge (MA), Harvard University Press.

Bronfenbrenner, U., et P.A. Morris (2006). «The bioecological model of human development », dans R.M. Lerner (dir.), Handbook of child psychology (6e éd., vol. 1), Hoboken (NJ), Wiley, p. 793-828.

Bruyère, E., et J. Garbarino (2010). "The ecological perspective on the human rights of children ", dans S.B. Kamerman, S. Phipps et A. Ben-Arieh (dir.), From child welfare to child well-being: An international perspective on knowledge in the service of policy paking, Londres, Springer, $\mathrm{p}$. 137-154.

Chaffin, M., L. Berliner, R. Block, T.C. Johnson, W. Friedrich, D. Louis, et J. F. Silovsky (2006). Report of the task force on children with sexual behavior problems. Récupéré de : http://www.atsa.com/sites/default/files/Report-TFCSBP.pdf

Chaffin, M., E. Letourneau et J. F. Silovsky (2002). "Adults, adolescents, and children who sexually abuse children: A developmental perspective », dans J.E.B. Myers, L. Berliner, J. Briere, C.T. Hendrix, C. Jenny et T.A. Reid (dir.), The APSAC handbook on child maltreatment ( $2^{\mathrm{e}}$ éd.), Thousand Oaks (CA), Sage, p. 205-232.

Cohen, J.A., A.P. Mannarino et E. Deblinger (2006). Treating trauma and traumatic grief in children and adolescents, New York (NY), Guilford Press. 
Deblinger, E., R.A. Steer et J. Lippman (1999). « Two-year follow-up study of cognitive behavioral therapy for sexually abused children suffering post-traumatic stress symptoms », Child Abuse \& Neglect, vol. 23, n 12, p. 1371-1378.

Dretzke, J., C. Davenport, E. Frew, J. Barlow, S. Stewart-Brown, S. Bayliss, ... C. Hyde (2009). "The clinical effectiveness of different parenting programmes for children with conduct problems: A systematic review of randomised controlled trials ", Child and Adolescent Psychiatry and Mental Health, vol. 3, $\mathrm{n}^{\circ} 7$.

Durand, C. (2006). La perception du phénomène de l'enfant agresseur sexuel chez les intervenants et intervenantes en protection au Centre jeunesse de Québec, Mémoire de maîtrise, Faculté des sciences sociales, Université Laval, Québec (QC).

Elkovitch, N., R.D. Latzman, D.J. Hansen, et M.F. Flood (2009). « Understanding child sexual behavior problems: A developmental psychopathology framework », Clinical Psychology Review, vol. 29, nº 7, p. 586-598.

Etgar, T., et H. Shulstain-Elrom (2010). «A combined therapy model (individual and family) for children with sexual behavior problems ", International Journal of Offender Therapy and Comparative Criminology, vol. 53, n 5, p. 574-595.

Evertsz, J., R. Miller, L. Bromfield, D. Higgins, R. Pratt, R. Noakes, ... J. Thompson (2010). Children with problem sexual behaviors and their families. Best interests case practice model. Specialist practice resource, Australia, Victorian Government of Human Services.

Friedrich, W.N., W. Davies, E. Fehrer et J. Wright (2003). « Sexual behavior problems in preteen children: Developmental, ecological, and behavioral correlates », Annals of the New York Academy of Sciences, vol. 989, p. 95-104.

Friedrich, W.N., A.J.L. Baker, R. Parker, M. Schneiderman, L. Gries et M. Archer (2005). « Youth with problematic sexualized behaviors in the child welfare system: A one-year longitudinal study ", Sexual Abuse: A Journal of Research and Treatment, vol. 17, n 4, p. 391-406.

Friedrich, W.N. (2007). Children with sexual behavior problems: Family-based, attachmentfocused therapy, New York (NY), Norton.

Gagnon, M. M., et M. Tourigny (2011). «Les comportements sexuels problématiques chez les enfants âgés de 12 ans et moins », dans M. Cyr, M. Hébert et M. Tourigny, L'agression sexuelle envers les enfants - Tome 1, Québec, Presses de l'Université du Québec, p. 333-362.

Gagnon, M. M., M. Tourigny et M. Lévesque (2008). «Enfants présentant des comportements sexuels problématiques : lignes directrices en matière d'évaluation et de traitement », Revue québécoise de psychologie, vol. 29, n³, p. 45-57.

Gil, E. (1993). «Family therapy », dans E. Gil et T. C. Johnson, Sexualized children: Assessment and treatment of sexualized children who molest, Rockville (MD), Launch Press, p. 275-302. 
Gil, E., et J.A. Shaw (2013). Working with children with sexual behavior problems, New York (NY), Guilford Press.

Greenberg, M.T., M.L. Speltz et M. Deklyen (1993). « The role of attachment in the development of early disruptive problems », Development and Psychopathology, vol. 5, nos 1-2, p. 191-213.

Hackett, S. (2011). " Children and young people with harmful sexual behaviours », dans C. Barter et D. Berridge (dir.), Children behaving badly? Peer violence between children and young people, Hoboken (NJ), Wiley-Blackwell, p. 121-135.

Hall, D., F. Mathews et J. Pearce (1998). « Factors associated with sexual behavior problems in young sexually, abused children », Child Abuse \& Neglect, vol. 22, n 10, p. 1045-1063.

Hall, D., F. Mathews, J. Pearce, N. Sarlo-McGarvey et D. Gavin (1996). The development of sexual behavior problems, South Pasadena (CA), Auteur.

Hélie, S., D. Turcotte, N. Trocmé et M. Tourigny (2012). Étude d'incidence québécoise sur les signalements évalués en protection de la jeunesse en 2008. Rapport final, Montréal, Centre jeunesse de Montréal - Institut universitaire.

Jaffé, P. D. (2011). «Enfants et adolescents auteurs d'abus sexuels - Pour une approche de la réhabilitation fondée sur des preuves scientifiques ", dans Conseil de l'Europe, La protection des enfants contre la violence sexuelle - Une approche globale, France, Conseil de l'Europe, p. 247-269.

Johnson, T.C. (2002). «Some considerations about sexual abuse in children with sexual behavior problems », Journal of Trauma \& Dissociation, vol. 3, n 4, p. 83-105.

Johnson, T.C. (2004). Helping children with sexual behaviour problems - A guidebook for parents and substitute caregivers ( $2^{\mathrm{e}}$ éd.), South Pasadena (CA), Auteur.

Johnson, T.C., et W.T. Aoki (1993). «Sexual behaviors of latency age children in residential treatment », Residential Treatment for Children \& Youth, vol. 11, n 1, p. 1-22.

Johnson, T.C. (2009). Helping children with sexual behaviour problems: A guidebook for professionals and caregivers ( $4^{\mathrm{e}}$ éd.), San Diego (CA), Institute on Violence, Abuse and Trauma.

Kazdin, A. E. (2005). Parent management training: Treatment for oppositional, aggressive, and antisocial behavior in children and adolescents, New York (NY), Oxford University Press.

Lepage, J., M. Tourigny, R. Pauzé, P. McDuff et M. Cyr (2010). "Comportements sexuels problématiques d'enfants pris en charge par les services québécois de protection de l'enfance : facteurs associés », Sexologies, vol. 19, n², p. 119-126. 
McGrath, R.J., G.F. Cumming, B.L. Burchard, S. Zeoli et L. Ellerby (2010). Current practices and emerging trends in sexual abuser management: The Safer Society 2009 North American survey, Brandon (VT), Safer Society Press.

Niec, L.N., J.M. Hemme, J.M. Yopp et E.V. Brestan (2005). « Parent-child interaction therapy: the rewards of challenges of a group format ", Cognitive and Behavioral Practice, vol. 12, $\mathrm{n}^{\circ}$ 1, $\mathrm{p}$. 113-125.

Nixon, R. (2002). « Treatment of behavior problems in preschoolers: A review of parent training programs », Clinical Psychology Review, vol. 22, n 4, p. 525-546.

Pevsner, R. (1982). «Group parent training versus individual family therapy: An outcome study », Journal of Behavior Therapy and Experimental Psychiatry, vol. 13, n² 2, p. 119-122.

Pithers, W.D., et A. Gray (1998). «The other half of the story: Children with sexual behavior problems ». Psychology, Public Policy and Law, vol. 4, ns 1/2, p. 200-217.

St-Amand, A., D.E. Bard et J.F. Silovsky (2008). « Meta-analysis of treatment for child sexual behavior problems: practice elements and outcomes », Child Maltreatment, vol. 13, n² 2, p. 145166.

St-Amand, A., M.-C. Saint-Jacques et J.F. Silovsky (2011). "Comprendre les enfants aux comportements sexuels problématiques et intervenir auprès d'eux. Bilan des connaissances », Revue canadienne de service social, vol. 28, n² 2, p. 225-253.

Sanders, M.R., W. Cann et C. Markie-Dadds (2003). «The Triple P-positive parenting program: A universal population-level approach to the prevention of child abuse ", Child Abuse Review, vol. $12, n^{\circ} 3$, p. 155-171.

Shevade, D., E. Norris et R. Swann (2011). «An exploration of therapists' reactions to working with children displaying sexually problematic behaviour: A thematic analytic study », Journal of Child Psychotherapy, vol. 37, n 1, p. 52-74.

Silovsky, J.F., et B.L. Bonner (2003). "Children with sexual behavior problems », dans T.H. Ollendick et C.S. Schroeder (dir.), Encyclopedia of clinical child and pediatric psychology, New York (NY), Kluwer Press, p. 589-591.

Silovsky, J.F., et L. Niec (2002). «Characteristics of young children with sexual behavior problems: A pilot study », Child Maltreatment, vol. 7, n 3, p. 187-197.

Silovsky, J.F., L. Niec, D.E. Bard et D. Hecht (2007). «Treatment for preschool children with interpersonal sexual behavior problems: A pilot study », Journal of Clinical Child and Adolescent Psychology, vol. 36, n 3, p. 378-391. 
Silovsky, J.F., et L.M. Swisher (2008). « Sexual development and sexual behavior problems », dans M.L. Wolraich, P. Dworkin, D. Drotar et E. Perrin, Evidence and practice in developmental and behavioral pediatrics: A comprehensive textbook, Philadelphie (PA), Elsevier, p. 805-824.

Silovsky, J.F., L.M. Swisher, J. Widdifield et L. Burris (2012). « Clinical considerations when children have problematic sexual behavior », dans P. Goodyear-Brown (dir.), Handbook of child sexual abuse: identification, assessment, and treatment, Hoboken (NJ), Wiley, p. 401-427.

Straus, S.E., W.S. Richardson, P. Glasziou et R.B. Haynes (2011). Evidence-based medicine: How to practice and teach it ( $4^{\mathrm{e}}$ éd). Édimbourg, Churchill Livingstone.

Szanto, L., J.S. Lyons et C. Kisiel (2012). « Childhood trauma experience and the expression of problematic sexual behavior in children and adolescents in state custody ", Residential Treatment for Children \& Youth, vol. 29, n³ 3, p. 231-249.

Vosmer, S., S. Hackett et M. Callanan (2009). " "Normal" and "inappropriate" childhood sexual behaviours: Findings from a Delphi study of professionals in the United Kingdom », Journal of Sexual Aggression, vol. 15, n 3, p. 275-288.

Webster-Stratton, C. (1984). «Randomized trial of two parent training programs for families with conduct disordered children », Journal of Consulting and Clinical Psychology, vol. 52, n 4, p. 666-678.

Webster-Stratton, C. (2005). «The incredible years: A training series for the prevention and treatment of conduct problems in young children », dans E. D. Hibbs et P. S. Jensen (dir.), Psychosocial treatments for child and adolescent disorders: Empirically based strategies for clinical practice ( $2^{\mathrm{e}}$ éd.), Washington (DC), American Psychological Association, p. 507-555. 